D. Kunth

Institut d'Astrophysique

Paris, France
W.L.W. Sargent

California Institute of Technology

Pasadena, USA

ABSTRACT. The Sculptor Region has been searched for quasar candidates: three IIIa-J objective prism plates from the UK Schmidt-telescope centred around the NGC55 and NGC300 galaxies have been used. 76 quasars have been found doubling the number of quasars previously known in this field. A test using Monte-Carlo simulations to reshuffle redshifts of the quasar sample shows that a simple eye examination of the simulated distributions produces as many quasar pairs or associations than in the original field. A further preliminary quantitative study using the autocorrelation function shows that quasar clustering is absent on any scale from 10 to $3500 \mathrm{Mpc}\left(H_{0}=100\right)$.

\title{
THE QUASAR SURVEY
}

Our initial goal has been a visual search of objective prism plates to discover bright quasars around NGC55 and NGC300. Further sensitive Ca absorption studies are planned to map metallic absorption in quasar spectra out to large radii around these nearby galaxies. Three plates have been taken at low dispersion and among hundreds of candidates we have spectroscopically observed about 170 objects and found 76 new quasars. Spectra were taken with the Las Campanas Intensified Reticon at the 100-inch Cassegrain spectrograph at low dispersion giving redshifts to an accuracy of \pm 0.01 except for few BAL QSOs for which line profile fitting is needed. A full account of the survey is given in Kunth and Sargent (1986).

A visual search is very subject to selection effects because it remains very hard to maintain the same search criteria all along the plate examination. The distribution of the objects is thus very clumpy and non-uniform as it is shown in Figure 1 and varies from field to field according to the quality of the plate. The distribution of magnitudes has a broad peak at about 18.8 similar to the distribution found by Osmer and Smith (1980). However while the limiting magnitudes are roughly the same, the number density of quasars found in this work largely exceeds ( 76 quasars in about $110 \mathrm{deg}^{2}$ ) the one reported by Arp (1984a) and Osmer and Smith (1980) for the same fields. This shows the limitations of the visual technique whenever aimed to discuss statis- 


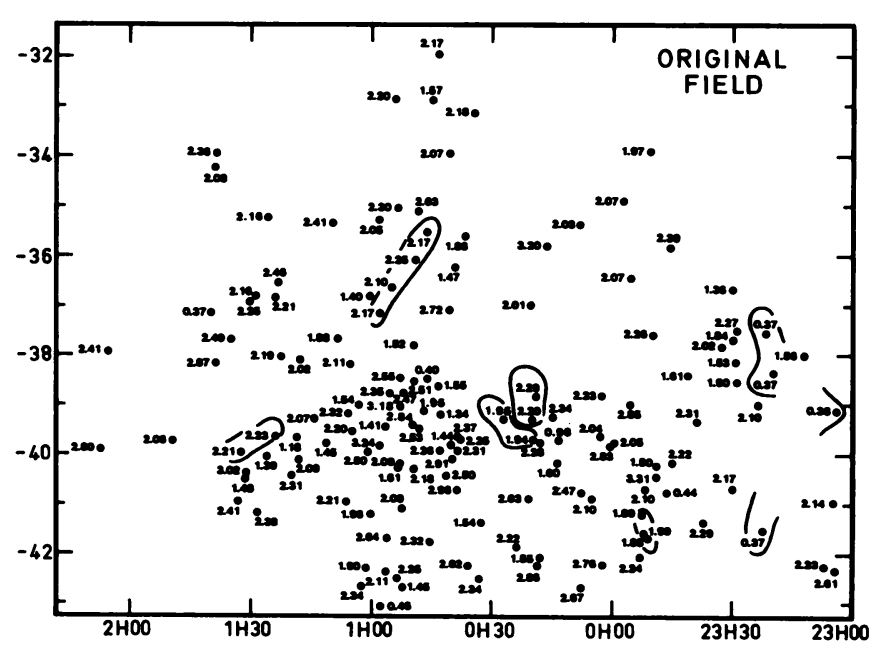

Figure 1 :

Plot of all known quasars -including those of this work- in the Sculptor Group found from OP Surveys. Declinations are in degrees. Some obvious pairs and groupings are encircled. tical distribution properties. Most of the selection effects have been quantified by Clowes (1981) and that reproduces quite well the observed continuum magnitudes at $5000 \AA$ against the equivalent widths of $L y \alpha+N V$ found in our sample. From a measure of the CIV equivalent widths we do not support Arp's suggestion (1984b) that the quasars in the Sculptor Group follow a well defined Baldwin relation. For this reason, and because of the non-uniformity of our survey, as well as the previous ones, we find no compelling evidence that quasars in the Sculptor Region have different distribution and intrinsic properties than field quasars.

\section{THE CLUSTERING}

Clustering at $z=2$ has been investigated by Osmer (1981) using a large set of objective prism plates centred in the Sculptor Group. We have performed such a preliminary analysis with our new set of quasars which about doubles the number of quasars over roughly $450 \mathrm{deg}^{2}$. Moreover the subareas around NGC55 and NGC300 were used to test clustering on small scales with higher sensitivity. This analysis has been performed by computing the observed autocorrelation function, corrected for cosmological effects and comparing it with the calculated autocorrelation function for a random distribution generated by Monte Carlo techniques. The details of the methods will be treated in a forthcoming paper. We found no clustering in all scales up to $3000 \mathrm{Mpc}$ (with $\mathrm{H}_{\mathrm{O}}=100 \mathrm{~km} \mathrm{sec}-1 \mathrm{Mpc}^{-1}$ ) but we still undergo a critical analysis of the method -using fake fields- before coming to firm conclusions about quasar clusterings.

The random distributions were obtained by preserving the same positions for the quasars on the sky but randomly shuffling the redshifts. The reason to keep the coordinates unchanged is that they are subject to the largest uncontrolled selection effects whereas 


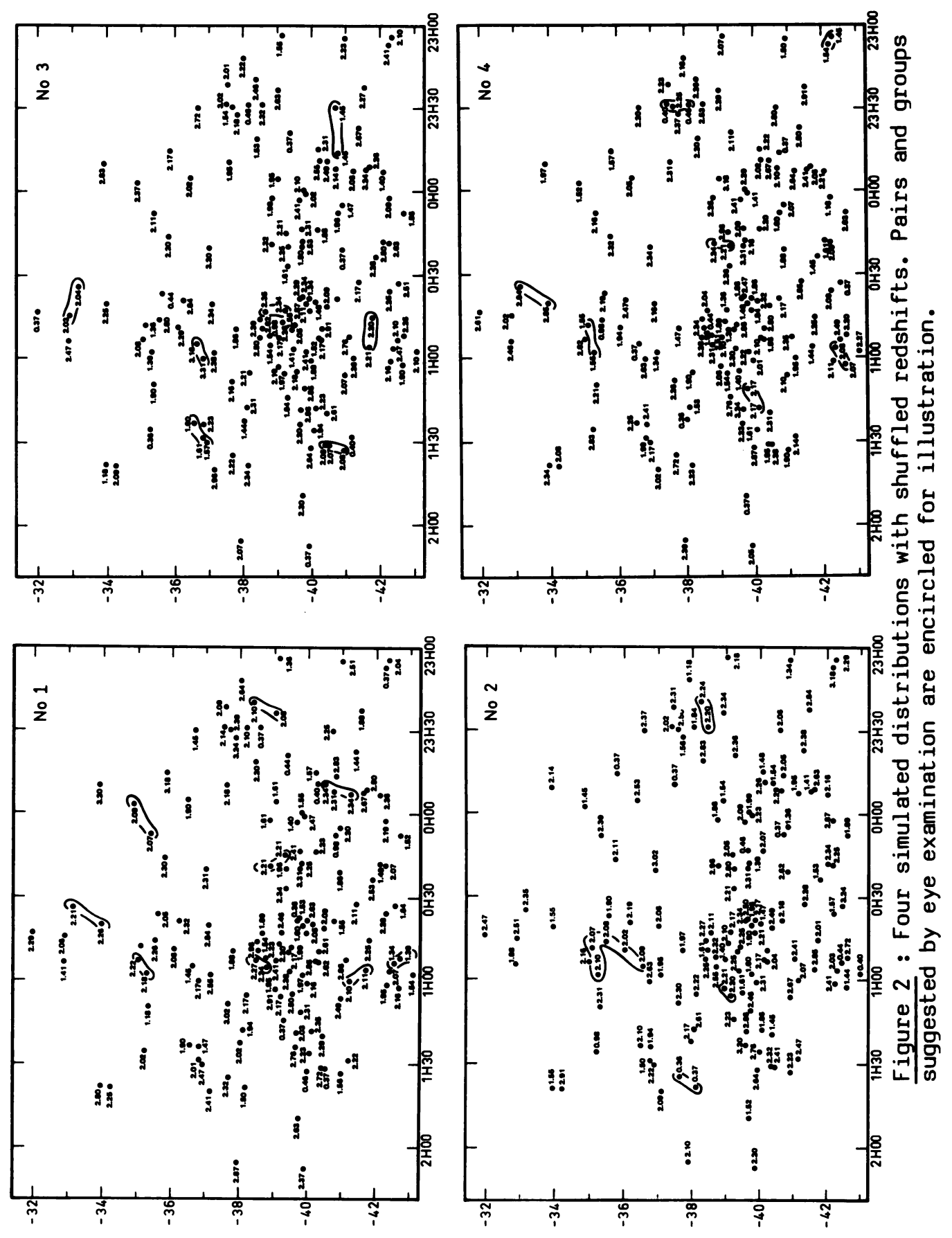


there is no reason to find any preferential redshift value within the range set by the objective prism technique. We have made the exercise of plotting some of the simulated distributions for illustration purpose and reproduced some of them (chosen at random) in Figure 2. It is interesting to see that as many pairs and groupings are found in the test fields than in the original one. This gives a visual illustration for the need to step on more quantifying estimates of the tendancy of quasars to cluster.

References

Arp, H., 1984a, Ap.J., 285,547

Arp, H., 1984b, Ap.J., 285,555

Clowes, R.G., 1981, M.N.R.A.S., 197,731

Kunth, D., Sargent, W.L.W., 1986, A.J., in press

Osmer, P., 1981, Ap.J., 247,762

Osmer, P., Smith, M.G., 1980, Ap.J. Suppl., $42,333$. 